phase. Perhaps unit staff who anticipated relatively more problems were more highly influenced by the success of the policy change.

\section{Conclusions}

Attitudes toward smoking and bans against smoking in the hospital perhaps reflect the complex interplay of social issues in the larger society. The values and meanings attached to smoking, particularly within a psychiatric setting, evoke a great deal of affect and create negative expectations about whether a smoking ban can be effective and can be tolerated by patients. Nonetheless, despite dire predictions, the smoking ban produced fewer negative effects and more positive effects than both medical and nursing staff had anticipated. Patients who smoked were able to reduce the actual amount of their smoking while in the hospital. They appear to have experienced significant increases in their self-esteem and sense of self-control as a result of temporary mastery of the urge to smoke.

This study suggests that hospitalized psychiatric patients, even those patients who are relatively more disturbed, are capable of making substantial, albeit temporary, changes in entrenched habits such as smoking.

\section{Acknowledgments}

The authors acknowledge the crucial participation in this study of the medical and nursing staff of the department of psychiatry at Providence Medical Center and of head nurses Heidi Grady and Sarah Sweeney.
References

1. Kottke TE, Hill C, Heitzig C, et al: Smoke-free hospitals: attitudes of patients, employees, and faculty. Minnesota Medicine 68:53-55, 1985

2. Enster VL, Wilner SI: Non-smoking policies in hospitals. Journal of Public Health Policy 6:197-203, 1985

3. Riffer J: Hospitals challenged to restrict smoking. Hospitals 56:20-24, 1982

4. Resnick MP, Gordon R, Bosworth EE: Evolution of smoking policies in Oregon psychiatric facilities, 1986-87. Hospital and Community Psychiatry 40:527529, 1989

5. Tamerin JS: The psychodynamics of quitting smoking in a group. American Journal of Psychiatry 129:101-107, 1972

6. Hughes JR, Hatsukami DK, Mitchell JE, et al: Prevalence of smoking among psychiatric outpatients. American Journal of Psychiatry 143:993-997, 1986

7. Moos RH: Ward Atmosphere Scale. Palo Alto, Calif, Consulting Psychologists Press, 1974

8. Moos RH: Evaluating Treatment Environments. New York, Wiley, 1974

\title{
Clinical Presentations of AIDS and HIV Infection in State Psychiatric Facilities
}

Ewald Horwath, M.D. Martin Kramer, M.D. Francine Cournos, M.D. Maureen Empfield, M.D. George Gewirtz, M.D.

The epidemiologic, neuropsychiatric, and medical data on AIDS and HIV infection that are relevant to state psychiatric facilities are reviewed. The epidemi-

Dr. Horwath is director, and Dr. Gewirtz is assistant director, of the community service inpatient unit at New York State Psychiatric Institute, 722 West 168th Street, New York, New York 10032. Dr. Kramer is chairman of the infection control committee at Kingsboro Psychiatric Center in Brooklyn, New York. Dr. Cournos is di- ologic data suggest that a langer than expected number of AIDS patients may be seen in these facilities. Patients who are severely disturbed and psycbotic may present to state bospitals with HIV encepbalopatby. In patients who are cbronically and severely ill, physical symptoms, including oral and cutaneous conditions, the HIV wasting syndrome, and lymphadenopatby, may provide early

rector of the Washington Heights Community Service of the New York State Psychiatric Institute. Dr. Empfield is clinical director of Creedmoor Psychiatric Center in Queens, New York. An earlier version of this paper was presented at the annual meeting of the American Psychiatric Association held May 7-13, 1988, in Montreal. clues to HIV infection. The early neuropsycbiatric and medical findings in HIV infection are discussed, and a clinical case is presented.

The epidemic of acquired immunodeficiency syndrome (AIDS) and human immunodeficiency virus (HIV) infection has already had a profound impact on the practice of medicine in the United States. As the epidemic continues to grow, it will undoubtedly have increasing impact on state psychiatric facilities as well. In this paper, we review epidemiologic, neuropsychiatric, and medical findings about AIDS and HIV infection that are relevant to state hospital settings. We also review our own experience in facilities operated by the New York State Office of Mental Health and discuss the implica- 
tions of our experience for mental health systems in other states.

\section{Epidemiology of AIDS}

The AIDS patient population in the United States is distinctly different from the general population in ethnic and racial composition. While blacks and Hispanics represented 12 percent and 6 percent, respectively, of the 1980 U.S. population (1), as of October 17, 1988 , they constituted 26 percent and 15 percent of patients with AIDS (2). The overrepresentation of blacks and Hispanics among AIDS patients is related to the high incidence within these groups of AIDS cases that are associated with intravenous (IV) drug abuse.

Poor and minority populations, including individuals who may be exposed to HIV infection through IV drug abuse, have traditionally turned to the public sector for their medical and psychiatric care. State psychiatric hospitals may therefore experience a large influx of patients with HIV infection as the AIDS epidemic grows. Patients with HIV infection may also enter state psychiatric facilities from the forensic system. A higher prevalence of HIV seropositivity has been noted among prisoners than among the general population (3), a difference that may be related to the overrepresentation among prisoners of past or current IV drug users.

The Centers for Disease Control (CDC) reported on January 9,1989 , that a total of 65,133 cases of AIDS had been detected in the United States (4). In New York City a total of 18,518 cases22 percent of all cases in the United States-were reported by the New York City Department of Health on January 12, 1989 (4). AIDS cases have shown an impressive regional concentration, but this distribution is expected to change as the proportion of cases among different risk groups changes. Fettner $(5)$ has projected that by 1991 New York City will have only 11.9 percent of all AIDS cases in the U.S. Psychiatric facilities throughout the nation need to be medically prepared for the impact of the projected redistribution of AIDS cases.

In acute medical settings, the early neuropsychiatric manifestations of AIDS are likely to include mood disturbance, mild cognitive dysfunctions, and anxiety symptoms (6-9). Such subtle disturbances are not likely to be presenting symptoms in state psychiatric facilities, where most patients are severely and chronically mentally ill. However, a patient who is experiencing severe behavioral disturbance and psychotic symptoms as a result of HIV infection may be admitted to a state hospital without staff s being aware that the patient is infected.

State psychiatric facilities are also more likely to see chronic mentally ill patients who have preexisting disorders such as schizophrenia and bipolar disorder and who have also acquired HIV infection (10). In these patients, the early subtle neuropsychiatric manifestations of HIV infection may be easily missed. Acutely ill psychiatric inpatients may have a higher rate of HIV infection than the general population. Goldwurm and associates (11) reported that seven of 97 patients ( 7.2 percent) hospitalized on a psychiatric ward were HIV seropositive. All seven patients had used intravenous drugs, and one also had a history of homosexuality. Three were hospitalized for schizophrenia, two for psychotic decompensation in the context of borderline personality disorder, and one for a suicide attempt.

However, data from a study at a Baltimore clinic for sexually transmitted diseases (12) indicated that a history of risk behavior may not be an adequate criterion for considering that individuals are at risk for HIV infection. Because patients presenting with acute psychiatric illnesses may suffer from thought disorder, paranoia, and impairments of memory, judgment, and insight, they may be even less reliable sources of this type of historical information. Mental health personnel are therefore not likely to know whether the individuals they treat have engaged in behavior that may have exposed them to HIV infection. To make an accurate diagnosis and provide appropriate treatment, clinicians should be aware of the early neuropsychiatric and medical signs and symptoms of HIV infection.

\section{Neuropsychiatric manifestations}

AIDS dementia complex. A syndrome of progressive dementia unrelated to any of the well-recognized opportunistic central nervous system (CNS) infections is increasingly being noted as a major cause of CNS dysfunction in AIDS patients. The syndrome is referred to as AIDS dementia complex, subacute encephalopathy or encephalitis, or AIDS-related dementia. It was first described by Snider and associates (13) in 1983 , and several subsequent reports have appeared $(14,15)$.

The clinical picture includes cognitive, motor, and behavioral signs and symptoms (14). Among the early cognitive symptoms are difficulty concentrating, impaired memory, and slowed-down thinking. Initial motor signs may include hand tremor, gait instability, or weakness of the legs. Common early behavioral manifestations are social withdrawal and apathy.

The syndrome progresses to a gross dementia characterized by disorientation, confusion, and mutism. Late in the illness, motor abnormalities may include paraplegia, weakness of the arms, lack of coordination, and urinary or bowel incontinence. Common behavioral manifestations in this stage of progression are agitation, paranoid delusions, and hallucinations.

Neurodiagnostic studies have been useful in establishing the diagnosis of AIDS dementia complex. Computerized tomography (CT) and magnetic resonance imaging (MRI) have demonstrated cerebral atrophy and white-matter abnormalities. Studies of cerebrospinal fluid (CSF) have shown nonspecific abnormalities, such as elevated CSF protein and mononuclear pleocytosis (15). In some cases identification of HIV has 
been possible by viral antigen detection or viral culture of the cerebrospinal fluid (16-18).

Mental health professionals should be aware that AIDS dementia complex may be the presenting or sole manifestation of HIV infection. Navia and Price (19) reviewed 112 patients with AIDS dementia complex over three years at Memorial SloanKettering Cancer Center in New York City. They identified 29 patients who developed this syndrome before or in the absence of major systemic opportunistic infections or neoplasm. The clinical manifestations included cognitive, motor, and behavioral signs and symptoms similar to those we have described. Behavioral disturbances were common and were among the prominent early signs in 11 patients. Of the 29 patients, six were medically well at the time of presentation and 23 had relatively mild manifestations of HIV infection, such as lymphadenopathy, weight loss, or malaise.

Based on these and other findings, Price and associates (20) estimated that early in the course of HIV infection, one-fourth of patients would manifest a subclinical or mild dementia demonstrable by psychological testing and as many as one-third would present with moderate to severe dementia.

Central nervous system dysfunction. Several other studies have indicated that patients with AIDS, AIDS-related complex (ARC), and HIV seropositivity may manifest early signs and symptoms of central nervous system dysfunction. Grant and associates (21) reported on a series of patients who underwent neuropsychological testing and MRI. Neuropsychological abnormalities-including impaired abstracting ability, learning difficulties, and slowed information processing-were found in 13 of 15 patients with AIDS, seven of 16 patients with ARC, seven of 13 who were HIV-seropositive, and only one of $11 \mathrm{HIV}$ seronegative controls. However, neuropsychological testing of patients in state hospitals may not reliably detect the subtle findings that have been associated with the early stages of HIV infection because many patients in state hospitals have significant, and often disabling, preexisting impairments in mental functions.

Cognitive and behavioral disturbances may be among the earliest manifestations of HIV infection and may lead to psychiatric referral before the onset of medical manifestations.

Grant and associates (21) used magnetic resonance imaging to examine the brains of a subgroup of the study patients. Nine of 13 AIDS patients and five of ten patients with ARC had evidence of MRI abnormalities. The most common abnormalities were sulcal and ventricular enlargement and bilateral patchy areas of high signal density in the subcortical white matter.

Acute psycbosis. Several reports suggest that early manifestations of HIV infection may include an acute psychosis. Halstead and others (22) described five cases of psychosis occurring in male homosexuals with HIV infection. Buhrich and associates (23) reported on three psychotic patients, one with AIDS and two with ARC, who showed no evidence of cognitive impairment. Jones and others (24) described a 27-year-old HIV-seropositive man who was apparently medically well but who presented with auditory hallucinations, thought blocking, and delusions of thought broadcasting.

Similarly, Maccario and Scharre (25) described a 35-year-old homosexual man with HIV seropositivity and cervical lymphadenopa- thy who presented with acute visual and olfactory hallucinations, paranoid ideation, and agitation. Navia and Price (19) reviewed 29 patients who presented with signs and symptoms of AIDS dementia complex. Agitated psychosis with paranoia and hallucinations was present initially in two of those patients.

These reports suggest that psychiatrists who evaluate patients who present with an acute psychotic illness should include early CNS manifestation of HIV infection in their differential diagnosis of acute psychosis. Although such presentations of the infection are relatively uncommon, they may represent an important clinical entity in state psychiatric hospitals and other settings such as psychiatric emergency rooms.

The accurate diagnosis of neuropsychiatric syndromes associated with HIV infection is quite diffcult. Cognitive and behavioral disturbances may be among the earliest manifestations of HIV infection and may lead to psychiatric referral before the onset of medical manifestations such as opportunistic infection or neoplasm. When the primary signs are behavioral, such as apathy and social withdrawal, the differential diagnosis should include such common psychiatric conditions as major affective disorder. When cognitive symptoms predominate, the range of causes of organic mental syndrome should be considered. In either case, the psychiatric evaluation should include a careful search for the characteristic cognitive, behavioral, and motor signs of AIDS dementia complex. Neurodiagnostic studies, such as CT or MRI scan, examination of the cerebrospinal fluid, and serologic testing for HIV antibodies can provide valuable diagnostic data.

\section{Other causes of}

neuropsychiatric disorder

In addition to the direct effects of HIV on the central nervous system, a number of structural and 
infectious processes related to impaired immunity may present as neuropsychiatric disorders. Tumors involving the brain include primary cerebral lymphoma and $\mathrm{Ka}$ posi's sarcoma. Opportunistic infections that affect the CNS include viruses such as herpes simplex, herpes zoster, cytomegalovirus, and papovavirus (progressive multifocal leukoencephalopathy); fungal infections such as cryptococcus and candida; protozoal infections such as toxoplasma; and bacterial infections such as mycobacterium avium-intracellulare. A careful evaluation for the presence of focal neurologic signs, symptoms of organic mental syndrome, and signs of CNS infection is indicated for patients suspected of having HIV infection.

\section{Early medical findings}

In an acute medical setting, neuropsychiatric manifestations of HIV infection may be the initial reason a patient comes to the attention of a psychiatrist. In state psychiatric facilities, however, early neuropsychiatric signs can easily be missed because subtle deficits in severely disturbed patients are difficult to evaluate.

In these patients, the most reliable early clues to the presence of HIV infection may be physical findings, particularly oral and cutaneous conditions. With the exception of Kaposi's sarcoma, most of the conditions we discuss may occur in young people who have no serious illness. However, the appearance of these conditions in severe forms and with unusually extensive distribution may herald the waning immunity associated with HIV infection.

Oral and cutaneous findings. $A$ variety of oral and cutaneous lesions have been described in association with HIV infection. Kaposi's sarcoma presents with an asymptomatic occasional nodule, a blue-gray hyperpigmented spot, or a papular rash. The diagnosis of Kaposi's sarcoma in a person under 60 years of age or in a person over 60 with laboratory evidence of HIV infection is suffcient for a diagnosis of AIDS (26). Seborrheic dermatitis, which may appear on the ears, scalp, face, and, less often, the anterior chest, axillae, and groin, has a higher frequency and is more extensive and inflammatory in people with AIDS or ARC than in patients who are not infected with HIV (27).

People with HIV infection have a higher frequency of herpes zoster, and the disease often presents with more severe pain, a larger number of vesicles, and a higher incidence of multidermatomal disease than would usually be seen (28). Severe cases of herpes simplex, especially the chronic perianal form, in HIV-infected persons have been described (29). Similarly, severe and extensive cases of dermatophytosis, xerosis, ichthyosis, oral candidiasis, oral hairy leukoplakia, and periodontitis may present in association with HIV infection (28-32).

Wasting syndrome. Progressive weight loss, which may be associated with fever or chronic diarrhea of more than one month's duration, is common in HIVinfected individuals. The condition thay result from the HIV infection directly or from associated opportunistic infections, neoplasm, or malnutrition. When seen in association with laboratory evidence of HIV infection, the wasting syndrome fits the CDC case definition of AIDS (26).

Lymphadenopatby. Lymphadenopathy is a common finding in patients infected with HIV. The syndrome of persistent generalized lymphadenopathy is defined as the presence of lymph nodes at least one centimeter in size in two or more extrainguinal sites for at least three months.

\section{Clinical case}

The following case of a patient with AIDS who was seen in a New York state psychiatric facility illustrates several of the clinical issues we have discussed.
Mrs. B, a 31 -year-old black unemployed divorced woman, was admitted for her first psychiatric hospitalization. She presented in a withdrawn psychotic state characterized by psychomotor retardation, paranoia, ideas of reference, auditory hallucinations, and poverty of thought. She admitted to cocaine use, but denied IV drug use or sexual contact with IV drug users. She also denied any history of medical problems.

An initial physical examination revealed a herpes simplex infection of the lip, a candida vaginal infection, oral candidiasis, and herpes zoster lesions on the left buttock. Twenty-five days after admission, she developed a high fever and was transferred to a general hospital, where a chest film was read as Pneumocystis carinii pneumonia. Further investigation revealed that a diagnosis of AIDS had been made eight months earlier during a previous episode of Pneumocystis carinii pneumonia. The patient herself continued to deny her diagnosis of AIDS.

This case illustrates several important points. First, a history of AIDS-related risk behaviors may be difficult or impossible to elicit from HIV-infected patients who are admitted to state hospitals. Second, a previous diagnosis of AIDS may be missed on an initial presentation of a severely ill psychiatric patient. Third, a psychotic illness may present for the first time in association with AIDS or HIV infection. And finally, oral, cutaneous, and other dermatologic findings may provide important early clues to the presence of HIV infection.

\section{Conclusions}

Populations served by state psychiatric facilities may be at greater risk for exposure to HIV infection and may be less likely to give adequate histories of AIDSrelated risk behaviors or previous medical illness. Some of the early signs of HIV infection, particularly the triad of cognitive, motor, 
and behavioral signs of AIDS dementia complex, may strongly suggest the etiology of a presenting neuropsychiatric disorder. For patients with severe and chronic psychiatric illness, however, such early neuropsychiatric signs may not be reliable indicators of HIV infection. In these patients, oral, cutaneous, and other physical findings can serve as early signs of HIV infection.

The clinical issues presented in this paper will have implications for the staffing, educational, and administrative needs of state psychiatric facilities. The permanent staff of those facilities will require training in recognizing the early manifestations of HIV infection and will need to depend more heavily on internists and other medical specialists to help assess and treat HIV-infected patients. Better relationships with general medical hospitals will need to be developed to ensure medical backup during acute medical crises. These and other management issues have been more fully discussed in a previous article (33).

As the regional distribution of AIDS cases changes, the experience of the staff of New York's state psychiatric facilities in recognizing the clinical presentations of HIV may provide important lessons for mental health professionals in regions that have not yet been as severely affected by the AIDS epidemic.

\section{References}

1. Bureau of the Census: Census of the Population, Vol 1: Characteristics of the Population. Washington, DC, US Department of Commerce, 1981

2. Centers for Disease Control statistics. AIDS 2:487-490, 1988

3. Centers for Disease Control: Human immunodeficiency virus infection in the United States: a review of current knowhedge. Morbidity and Mortality Weekly Report 36(suppl 6):1S-48S, 1987

4. AIDS Surveillance Unit: AIDS Surveillance Update. New York City, Department of Health, Jan 25, 1989

5. Fettner AG: The discovery of AIDS: perspectives from a medical journalist, in AIDS and Other Manifestations of
HIV Infection. Edited by Wormser GP. Park Ridge, NJ, Noyes, 1987

6. Perry SW, Tross S: Psychiatric problems of AIDS inpatients at the New York Hospital: preliminary report. Public Health Reports 99:200-205, 1984

7. Holland JC, Tross S: The psychosocial and neuropsychiatric sequelae of the acquired immunodeficiency syndrome and related disorders. Annals of Internal Medicine 103:760-764, 1985

8. Perry S, Jacobsen P: Neuropsychiatric manifestations of AIDS-spectrum disorders. Hospital and Community Psychiatry 37:135-142, 1986

9. Faulstich ME: Psychiatric aspects of AIDS. American Journal of Psychiatry 144:551-556, 1987

10. Gewirtz G, Horwath E, Cournos F, et al: Patients at risk for HIV (ltr). Hospital and Community Psychiatry 39:1311-1312, 1988

11. Goldwurm GF, Zamparetti M, Caggese L, et al: Prevalence of HIV antibody tests (HIVab+) among the patients of a psychiatric ward. Presented at the International Conference on AIDS, Stockholm, Sweden, June 1988

12. Quinn TC, Glasser D, Cannon RO, et al: Human immunodeficiency virus infection among patients attending clinics for sexually transmitted diseases. New England Journal of Medicine 318:197-203, 1988

13. Snider WD, Simpson DM, Nielsen S, et al: Neurological complications of acquired immune deficiency syndrome: analysis of 50 parients. Archives of Neurology 14:403-418, 1983

14. Navia BA, Jordan BD, Price RW: The AIDS dementia complex, I: clinical features. Annals of Neurology 19:517524,1980

15. Navia BA, Cho ES, Petito CK, et al: The AIDS dementia complex, II: neuropathology. Annals of Neurology 19:525-535, 1986

16. Ho DD, Rota TR, Schooley RT, et al: Isolation of HTLV-III from cerebrospinal fluid and neural tissue of patients with neurologic syndromes related to the acquired immunodeficiency syndrome. New England Journal of Medicine 313:1493-1497, 1985

17. Levy JA, Shimabukuro J, Hollander $\mathrm{H}$, et al: Isolation of AIDS-associated retroviruses from cerebrospinal fluid and brain of patients with neurological symptoms. Lancet 2:586-588, 1985

18. Pumarola-Sune T, Navia BA, CordonCardo C, et al: HIV antigen in the brains of patients with the AIDS dementia complex. Annals of Neurology 21:490-496, 1987

19. Navia BA, Price RW: The acquired immunodeficiency syndrome dementia complex as the presenting or sole manifestation of human immunodeficiency virus infection. Archives of Neurology 44:65-69, 1987

20. Price RW, Sidtis JJ, Navia BA, et al: The AIDS dementia complex, in AIDS and the Nervous System. Edited by Rosenblum ML. New York, Raven, 1988

21. Grant I, Atkinson JH, Hesselink JR, et al: Evidence for early central nervous system involvement in the acquired immunodeficiency syndrome (AIDS) and other human immunodeficiency virus (HIV) infections. Annals of Internal Medicine 107:828-836, 1987

22. Halstead S, Riccio M, Harlow P, et al: Psychosis associated with HIV infection. British Journal of Psychiatry 153:618-623, 1988

23. Buhrich N, Cooper DA, Freed E: HIV infection associated with symptoms indistinguishable from functional psychosis. British Journal of Psychiatry 152:649-653, 1988

24. Jones GH, Kelly CL, Davies JA: HIV and the onset of schizophrenia. Lancet 1:982, 1987

25. Maccario M, Scharre DW: HIV and acute onset of psychosis. Lancet 2:342, 1987

26. Centers for Disease Control: Revision of the CDC surveillance case definition for acquired immunodeficiency syndrome. Morbidity and Mortality Weekly Report 36(suppl1):1S-15S, 1987

27. Mathes BM, Douglas MC: Seborrheic dermatitis in patients with acquired immunodeficiency syndrome. Journal of the American Academy of Dermatology 13:947-951, 1985

28. Kaplan MH, Sadick N, McNutt NS: Dermatologic findings and manifestations of acquired immunodeficiency syndrome (AIDS). Journal of the American Academy of Dermatology 16:485S06, 1987

29. Goodman DS, Teplitz ED, Wishner A, et al: Prevalence of cutaneous disease in patients with acquired immunodeficiency syndrome (AIDS) or AIDSrelated complex. Journal of the American Academy of Dermatology 17:210 220, 1987

30. Feigal DW, Abrams DI, Greenspan JS, et al: Oral candidiasis and progression to initial AIDS-associated opportunistic infections. Presented at the International Conference on AIDS, Washington, DC, June 1987

31. Greenspan D, Greenspan JS, Hearst NG, et al: Relation of oral hairy leukoplakia to infection with the human immunodeficiency virus and the risk of developing AIDS. Journal of Infectious Diseases 155:475-481, 1987

32. Winkler JR, Grass M, Murray PA: Periodontal disease in HIV-infected male homosexuals. Presented at the International Conference on AIDS, Washington, DC, June 1987

33. Cournos F, Empfield M, Horwath E, et al: The management of HIV infection in state hospitals. Hospital and Community Psychiatry 40:153-157, 1989 